\title{
Frequency sensitive moment tensor inversion for light to moderate magnitude earthquakes in eastern Africa
}

\author{
A. Barth, ${ }^{1,2}$ F. Wenzel, ${ }^{2}$ and D. Giardini ${ }^{3}$ \\ Received 27 April 2007; revised 18 June 2007; accepted 25 June 2007; published 7 August 2007.
}

[1] We provide a procedure for the routine determination of moment tensors from earthquakes with magnitudes as low as $M_{W} 4.4$ using data recorded by only a few permanent seismic stations at regional to teleseismic distances. Waveforms are inverted for automatically determined frequency pass-bands that depend on source-receiver locations as well as the earthquake magnitude. Inversion results are stable against small variations in the frequency band and provide low data variances, i.e., a good fit between observed and modelled waveform traces. The total frequency band used for our procedure ranges from $10 \mathrm{mHz}$ to $29 \mathrm{mHz}$ (periods of $35 \mathrm{~s}$ to $100 \mathrm{~s}$ ). This enables us to determine focal mechanisms for earthquakes that were not derived previously by routine procedures of CMT or other agencies. As a case study, we determine focal mechanism solutions of 38 light to moderate magnitude earthquakes in eastern Africa between 1995 and 2002. Citation: Barth, A., F. Wenzel, and D. Giardini (2007), Frequency sensitive moment tensor inversion for light to moderate magnitude earthquakes in eastern Africa, Geophys. Res. Lett., 34, L15302, doi:10.1029/ 2007GL030359.

\section{Introduction}

[2] Earthquake source parameters reveal valuable information on seismotectonics and earthquake hazard. Thus in intraplate regions of only moderate seismicity low magnitude earthquake focal mechanisms can give important insights into active tectonics [Fan and Wallace, 1991].

[3] Harvard CMT-solutions are calculated automatically inverting long-period body waves and surface waves using periods of $T>45 \mathrm{~s}$ respective $T>135 \mathrm{~s}$, recorded by permanent stations of the global seismic network in teleseismic distances [Dziewonski et al., 1987]. On a global scale the level of completeness for Harvard CMT-solutions is approximately $M_{W} 5.5$ and slightly lower for some regions depending on the present station distribution [Arvidsson and Ekström, 1998]. To lower this magnitude threshold, regional station networks have been used to calculate source mechanisms by moment tensor inversion for $M_{W}<5.0$ [e.g., Pinar et al., 2003; Stich et al., 2003]. Stich et al. [2003] used waveform data of light to moderate magnitude earthquakes recorded in local to regional dis-

\footnotetext{
${ }^{1}$ Heidelberg Academy of Sciences and Humanities, Heidelberg, Germany.

${ }^{2}$ Geophysical Institute, University of Karlsruhe, Karlsruhe, Germany.

${ }^{3}$ Swiss Seismological Service, Institute of Geophysics, ETH Zürich, Zürich, Switzerland.
}

Copyright 2007 by the American Geophysical Union. 0094-8276/07/2007GL030359\$05.00 tances up to $1000 \mathrm{~km}$. They inverted periods ranging from $20 \mathrm{~s}$ to $50 \mathrm{~s}$ and $15 \mathrm{~s}$ to $35 \mathrm{~s}$ for events with $M_{W}>4.0$ and $M_{W}<4.0$, respectively. Source-relevant teleseismic recordings with a high signal-to-noise ratio of light to moderate events $\left(M_{W} 4.0-5.9\right)$ are obtained only in a limited frequency band that is restricted by seismic noise at the station-site [Stutzmann et al., 2000], the intrinsic attenuation of waveenergy [e.g., Anderson and Hart, 1978] and heterogeneities in the surface wave phase velocity pattern [Arvidsson and Ekström, 1998]. Dependent on the earthquake magnitude and source-receiver distances, the inverted frequency band of waveform data has to be chosen adequately [Braunmiller et al., 2002].

[4] In this work, we present a semi-automatic and frequency sensitive moment tensor inversion that allows to determine low magnitude earthquake focal mechanisms for regions where only sparse data are available. For each event the procedure automatically selects high quality waveform data and adjusts the frequency band to the individual setting, i.e., source-receiver locations and the earthquake magnitude [Barth, 2007]. As a case study, we analyse dependencies of the inversion result on hypocentral depth and frequency pass-band for light to moderate magnitude earthquakes in eastern Africa (Figure 1).

\section{Frequency Sensitive Moment Tensor Inversion}

[5] We use a damped least squares moment tensor inversion in frequency-domain to match the spectral phase and amplitude radiation pattern [Giardini, 1992; Bernardi et al., 2004]. We allow re-alignment in time-domain to account for traveltime delays apparent on intermediate periods [Giardini, 1992; Arvidsson and Ekström, 1998]. These time-shifts are restricted to a quarter of the smallest inverted period to prevent polarity changing phase-shifts. The amount of time-shift does improve the waveform fit but does not significantly change the final focal mechanism [Arvidsson and Ekström, 1998]. Identical time-shifts for the radial and vertical component are applied to fit vertical polarised waves (Rayleigh- and $\mathrm{P} / \mathrm{SV}$-waves) as an individual time-adjustment is allowed for the transverse component containing horizontal polarised waves (Loveand SH-waves). The inversion has a limited depth resolution, since long and intermediate period surface waves excitation functions do not vary much with source depth [Giardini, 1992]. Hence, we use discrete crustal hypocentral depths $(6,10,14,18,25,33$ and for some events $42 \mathrm{~km})$ [Bernardi et al., 2004]. Synthetics are generated by normal mode summation [Woodhouse, 1988] for the PREM 1D earth model [Dziewonski and Anderson, 1981] that makes the inversion routine easily transferable to regions world- 


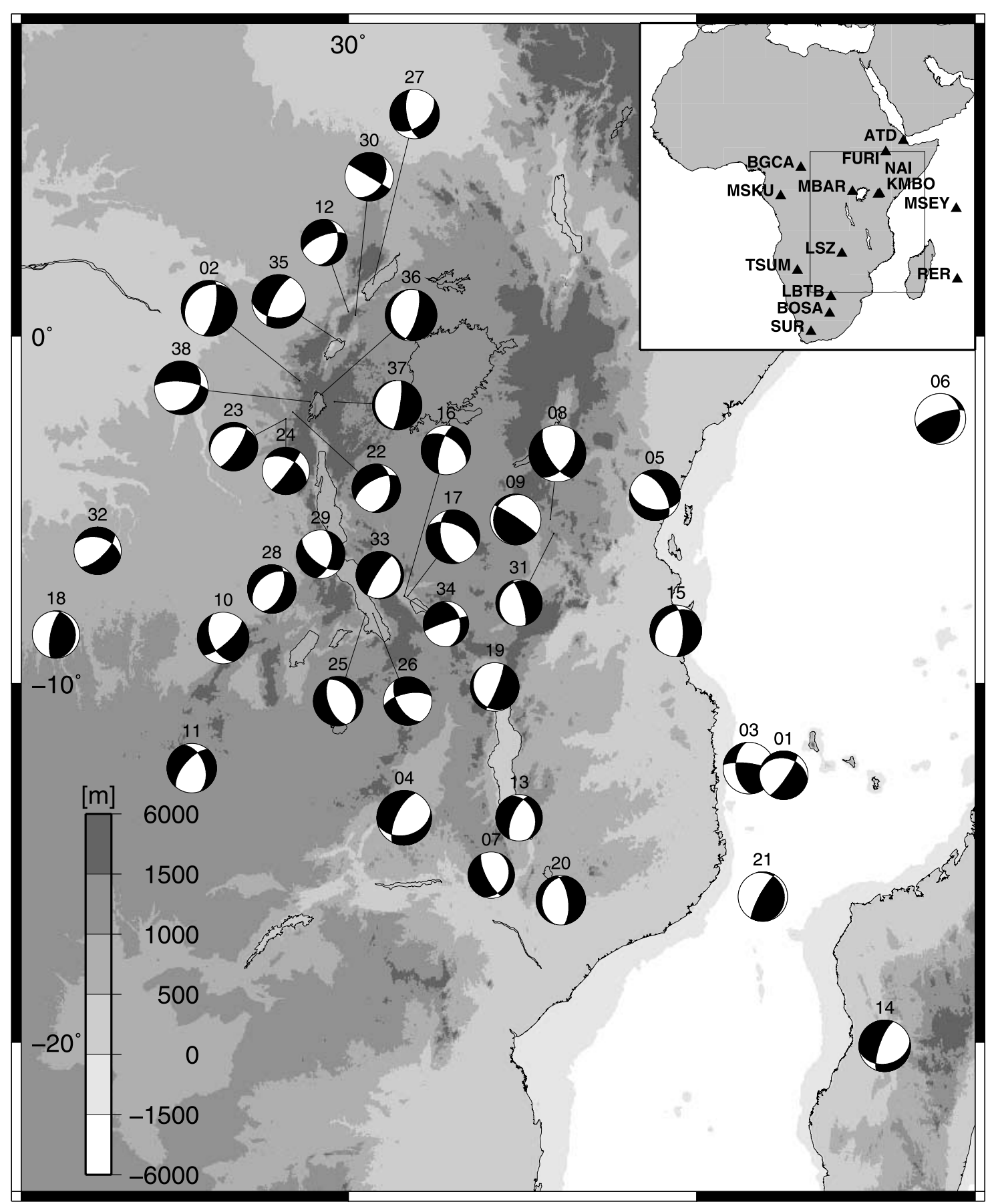

Figure 1. Topographic map of eastern Africa showing the 38 focal mechanisms calculated. For numbering see Table 1. Used seismic stations are plotted in the inset.

wide. However, this restricts the inversion to long-period waves, that are less influenced by structural heterogeneities than short periods [Larson and Ekström, 2001]. Beside this short-period limit, the usable waveform data are also limited for long-period waves, since low magnitude earthquakes provide amplitudes higher than the seismic noise level only at waves of rather short periods when recorded at teleseismic distances. These two limits restrict the data used in this study to frequencies from $10 \mathrm{mHz}$ to $29 \mathrm{mHz}$ (periods $35-100 \mathrm{~s})$.
[6] The data processing follows a strict scheme and includes three steps: 1 . Determination of an initial data set $N_{\text {init }}$ (for an initial and representative frequency range $\left.F_{\text {init }}\right) ; 2$. Extraction of the final data set $N_{\text {fin }}$ in dependence on hypocentral depth and inverted frequency range; 3 . Frequency sensitive analysis for the final data set to determine the optimum hypocentral depth and the final frequency range $F_{f i n}$.

[7] (1) Due to site dependent seismic noise and crustal heterogeneities along the travel paths of the recorded waves, 


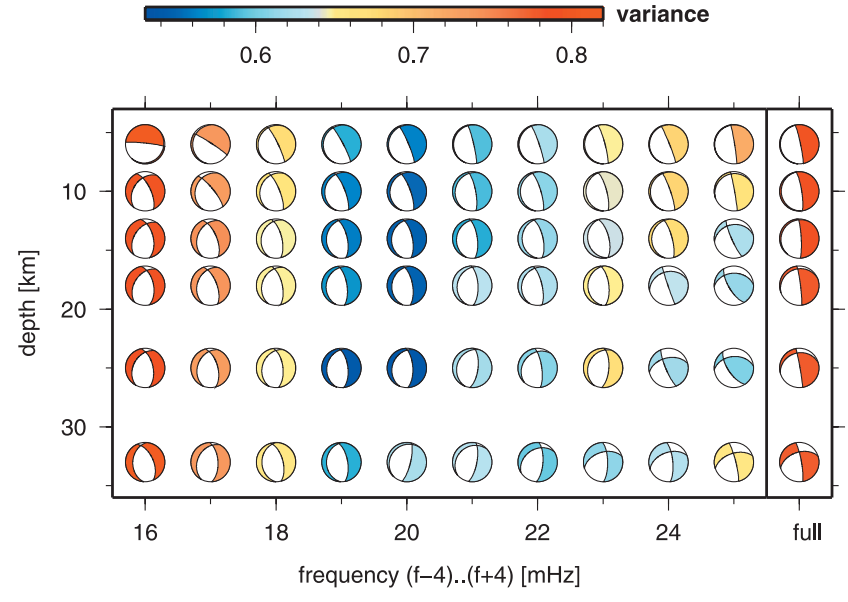

Figure 2. Frequency sensitive moment tensor inversion for event number 20. Inversion results of the data set $N_{\text {fin }}$ are shown for different combinations of discrete hypocentral depths and $8 \mathrm{mHz}$ wide frequency pass-bands within the frequency range $F_{\text {init }}$, shaded by variance $\sigma$. Indicated frequencies correspond to the mid-frequency within the pass-band. The right column gives the inversion results for the full frequency range from $10-29 \mathrm{mHz}$.

some waveforms are not usable for the moment tensor inversion. Thus at first, each available single seismometer component is reviewed by moment tensor inversion whether its waveform can be explained by an arbitrary source mechanism and the 1D earth model. The inversion for the deviatoric moment tensor is performed for a sliding $8 \mathrm{mHz}$ wide frequency band between $8 \mathrm{mHz}$ and $29 \mathrm{mHz}$ and a constant hypocentral depth given by Engdahl et al. [1998]. For each frequency band we calculate the variance $\sigma$ between observed $\mathbf{d}$ and synthetic waveforms $\mathbf{s}$ in the frequency domain:

$$
\sigma=\frac{(\mathbf{d}-\mathbf{s})^{2}}{\mathbf{d}^{2}},
$$

to choose the initial data set $N_{\text {init }}$ and frequency range $F_{\text {init }}$ such that each trace has a $\sigma$ below 0.5 [Barth, 2007].

[8] (2) Next we reduce the subset $N_{\text {init }}$ to the set of waveforms $N_{f i n}$ that can be explained by a common moment tensor: One by one the waveform explained worst is excluded from the subset $N_{\text {init }}$ and the inversion is performed again. This step is repeated for a sliding frequency band within the frequency range $F_{\text {init }}$ in combination with discrete crustal source depths. The iterative process reduces $N_{\text {init }}$ to an individual data set for each depth-frequency combination.

[9] (3) Finally, the frequency sensitive inversion is performed for the final data set $N_{f i n}$ and applied for the discrete source depths and the frequency range $F_{\text {init }}$ by sliding frequency bands. The depth-frequency pair with the lowest variance $\sigma$ and consistent focal planes for neighbouring frequency bands is selected and manually controlled. The chosen band-width is extended to the widest range of similar focal mechanisms, thus obtaining the minimum variance frequency range $F_{\text {fin }}$ of a stable source mechanism (Figure 2). To define similarity of two focal mechanisms, we use the average difference in orientation of the principal axes of the moment tensor $|\Delta A x|<30^{\circ}$ [Bernardi et al., 2004]. At last the inversion of $N_{f i n}$ and $F_{f i n}$ for the discrete source depths determines the hypocentral depth with the lowest variance $\sigma$.

[10] A representative example is event number 20 that occurred on 4 January 2000 on the southernmost part of the East African Rift in Mozambique $\left(36.1^{\circ} \mathrm{E} / 16.1^{\circ} \mathrm{S}\right.$, see Figure 1). Figure 2 shows the result of the third and final step of the processing scheme described above. Hereby, the dependency of the inversion result on hypocentral depth and frequency pass-band is analysed over the individual frequency range $F_{\text {init }}=\{12 \mathrm{mHz} . .29 \mathrm{mHz}\}$. The minimum variance frequency band $F_{\text {fin }}$, for which data can be explained best, ranges from $15 \mathrm{mHz}$ to $24 \mathrm{mHz}$ for a hypocentral depth of $25 \mathrm{~km}$. Although the focal mechanism remains stable for a wide frequency range, the variance $\sigma$ increases towards higher as well as lower frequencies. In Figure 3 we present the detailed inversion result for the optimum source depth of $25 \mathrm{~km}$. Finally, we use eight waveform traces of four seismic stations to determine the moment tensor. Source-receiver distances are between $2000 \mathrm{~km}$ and $3000 \mathrm{~km}$. The seismic moment is determined to $1.66 \times 10^{16} \mathrm{Nm}\left(M_{W}\right.$ 4.8). Surface wave-trains on both radial and transverse components are fitted very well in phase and amplitude with a variance $\sigma=0.60$. Detailed figures for other
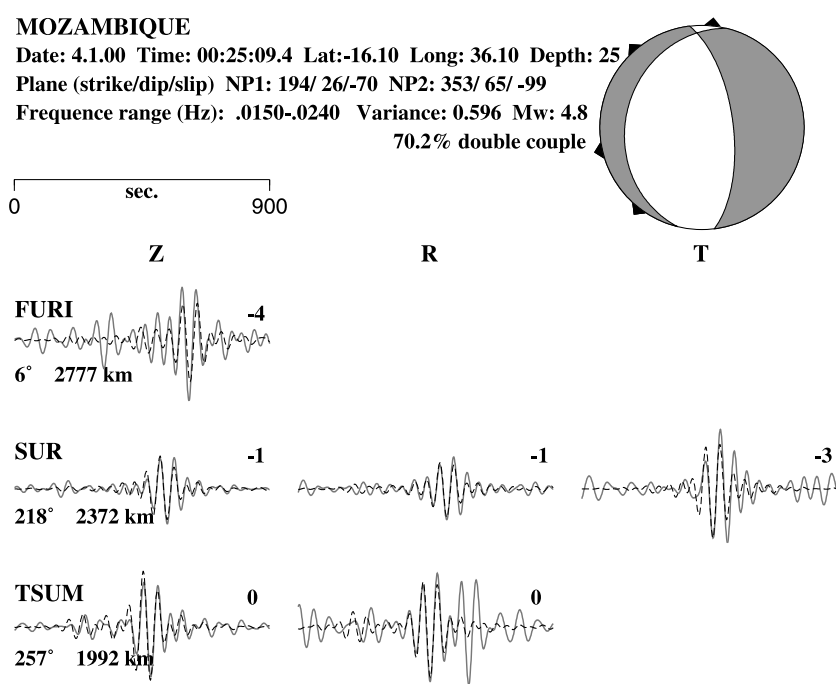

BGCA

$319^{\circ} 3051 \mathrm{~km}$

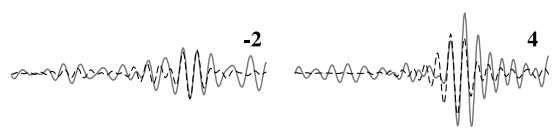

Figure 3. Final result of the moment tensor inversion for the frequency range $F_{f i n}$ for event number 20 . Triangles around the focal mechanism indicate station azimuth. The inverted waveform traces $N_{\text {fin }}$ on the vertical, radial and transverse component $(\mathrm{Z}, \mathrm{R}, \mathrm{T})$ are plotted as solid lines, synthetics as dashed lines. Station name and time shift [s] is given above each vertical trace, azimuth and epicentral distance below. Amplitudes are plotted normalised to the maximum of each station. 
Table 1. Results of the Frequency Sensitive Moment Tensor Inversion ${ }^{\mathrm{a}}$

\begin{tabular}{|c|c|c|c|c|c|c|c|c|c|c|c|c|c|}
\hline $\mathrm{Nr}$ & Date & Time & Lat & Lon & $\mathrm{D}$ & $M_{W}$ & $\phi$ & $\delta$ & $\lambda$ & $N_{\text {fin }}$ & $\sigma$ & $F_{\text {fin }}$ & $\mathrm{Q}$ \\
\hline 1 & $1995-04-27$ & $02: 32: 21.0$ & -12.6 & 42.5 & 25 & 4.7 & 283 & 30 & -24 & 6 & 0.43 & $18-29$ & B \\
\hline 2 & $1995-04-29$ & $11: 50: 53.9$ & -1.3 & 28.6 & 6 & 5.4 & 229 & 22 & -55 & 6 & 0.55 & $20-29$ & $\mathrm{C}$ \\
\hline 3 & 1995-07-20 & $05: 08: 28.4$ & -12.4 & 41.5 & 18 & 5.0 & 174 & 48 & 160 & 6 & 0.19 & $19-29$ & B \\
\hline 4 & 1995-11-12 & 19:00:09.8 & -13.8 & 31.6 & 33 & 5.3 & 76 & 36 & -45 & 6 & 0.20 & $17-29$ & B \\
\hline 5 & 1995-12-08 & $23: 40: 51.8$ & -4.6 & 38.8 & 18 & 4.9 & 102 & 43 & -128 & 8 & 0.47 & $19-29$ & B \\
\hline 6 & 1996-02-29 & $07: 14: 18.7$ & -2.4 & 47.0 & 10 & 4.9 & 19 & 26 & 42 & 7 & 0.33 & $20-29$ & $\mathrm{C}$ \\
\hline 7 & 1996-08-30 & $06: 58: 45.2$ & -15.4 & 34.1 & 10 & 4.5 & 21 & 27 & -46 & 9 & 0.56 & $21-29$ & B \\
\hline 8 & $1996-12-21$ & $08: 34: 06.2$ & -5.3 & 35.8 & 25 & 5.5 & 33 & 49 & -37 & 9 & 0.31 & $10-22$ & B \\
\hline 9 & $1997-02-23$ & $01: 39: 34.9$ & -5.3 & 34.8 & 10 & 4.9 & 190 & 14 & 155 & 10 & 0.44 & $15-29$ & A \\
\hline 10 & 1997-04-15 & $19: 04: 29.2$ & -8.7 & 26.4 & 18 & 5.0 & 156 & 49 & -154 & 7 & 0.71 & $17-27$ & B \\
\hline 11 & 1998-04-12 & $10: 49: 00.8$ & -12.4 & 25.5 & 33 & 4.8 & 340 & 46 & -141 & 7 & 0.31 & $17-27$ & B \\
\hline 12 & $1998-08-15$ & $17: 29: 19.0$ & 0.7 & 30.0 & 10 & 4.5 & 13 & 41 & -138 & 8 & 0.49 & $19-29$ & B \\
\hline 13 & 1998-08-24 & $12: 12: 11.1$ & -13.8 & 34.9 & 10 & 4.5 & 339 & 33 & -129 & 6 & 0.44 & $20-29$ & $\mathrm{C}$ \\
\hline 14 & 1998-09-22 & $19: 27: 35.2$ & -20.1 & 45.4 & 33 & 5.0 & 78 & 30 & -35 & 19 & 0.29 & $18-29$ & A \\
\hline 15 & 1999-04-06 & $04: 16: 47.0$ & -8.5 & 39.4 & 14 & 5.0 & 223 & 31 & -50 & 10 & 0.33 & $15-28$ & A \\
\hline 16 & 1999-05-07 & $02: 10: 45.1$ & -7.5 & 31.6 & 25 & 4.8 & 305 & 48 & -152 & 16 & 0.53 & $19-29$ & A \\
\hline 17 & 1999-05-07 & $14: 07: 32.9$ & -7.5 & 31.7 & 25 & 5.2 & 294 & 52 & -130 & 16 & 0.42 & $13-24$ & A \\
\hline 18 & 1999-08-06 & $17: 22: 48.8$ & -8.6 & 21.6 & 10 & 4.6 & 354 & 19 & 73 & 7 & 0.47 & $17-29$ & B \\
\hline 19 & 1999-09-01 & $04: 07: 56.5$ & -10.1 & 34.2 & 10 & 4.7 & 147 & 16 & -144 & 7 & 0.38 & $18-29$ & B \\
\hline 20 & 2000-01-04 & $00: 25: 09.4$ & -16.1 & 36.1 & 25 & 4.8 & 194 & 26 & -70 & 8 & 0.60 & $15-24$ & B \\
\hline 21 & 2000-01-14 & $03: 32: 44.2$ & -16.0 & 41.9 & 6 & 4.8 & 347 & 14 & 49 & 6 & 0.32 & $21-29$ & $\mathrm{C}$ \\
\hline 22 & 2000-03-02 & $02: 44: 55.5$ & -2.2 & 28.4 & 6 & 4.7 & 9 & 42 & -133 & 11 & 0.48 & $19-29$ & A \\
\hline 23 & 2000-03-02 & $04: 29: 50.2$ & -2.4 & 28.2 & 25 & 4.7 & 33 & 75 & -103 & 6 & 0.44 & $17-28$ & B \\
\hline 24 & 2000-03-03 & $05: 03: 22.3$ & -2.4 & 28.2 & 33 & 4.6 & 37 & 83 & -137 & 7 & 0.56 & $17-29$ & B \\
\hline 25 & $2000-10-05$ & $23: 08: 35.9$ & -8.0 & 30.5 & 25 & 4.8 & 161 & 57 & -84 & 12 & 0.39 & $21-29$ & B \\
\hline 26 & 2000-10-07 & $01: 39: 10.4$ & -8.0 & 30.7 & 25 & 4.7 & 149 & 56 & -32 & 10 & 0.34 & $21-29$ & B \\
\hline 27 & $2000-10-23$ & $12: 02: 14.7$ & 1.5 & 30.7 & 25 & 4.8 & 52 & 48 & -37 & 10 & 0.52 & $19-29$ & A \\
\hline 28 & $2000-12-02$ & $04: 16: 43.0$ & -7.3 & 27.8 & 18 & 4.7 & 230 & 40 & -68 & 10 & 0.35 & $16-24$ & B \\
\hline 29 & $2000-12-15$ & $10: 01: 22.8$ & -5.5 & 29.4 & 33 & 4.7 & 12 & 52 & -40 & 14 & 0.36 & $21-29$ & B \\
\hline 30 & 2001-01-31 & $19: 15: 29.2$ & 0.6 & 30.2 & 33 & 4.7 & 32 & 40 & -179 & 9 & 0.41 & $18-29$ & B \\
\hline 31 & $2001-03-25$ & $18: 54: 14.2$ & -5.7 & 35.9 & 33 & 4.5 & 202 & 17 & -53 & 6 & 0.43 & $15-28$ & B \\
\hline 32 & 2001-04-17 & 04:39:03.4 & -6.2 & 22.8 & 33 & 4.6 & 287 & 43 & -37 & 12 & 0.53 & $21-29$ & B \\
\hline 33 & 2001-07-13 & $19: 24: 02.4$ & -6.9 & 30.9 & 42 & 4.6 & 12 & 12 & -110 & 10 & 0.29 & $21-29$ & B \\
\hline 34 & 2001-09-18 & 11:01:02.1 & -7.5 & 31.6 & 10 & 4.4 & 350 & 37 & -169 & 11 & 0.56 & $19-29$ & A \\
\hline 35 & 2002-01-04 & $13: 02: 21.2$ & -0.2 & 29.8 & 10 & 5.2 & 90 & 38 & -32 & 6 & 0.17 & $15-26$ & B \\
\hline 36 & 2002-01-17 & $20: 01: 30.7$ & -1.7 & 29.2 & 25 & 5.0 & 16 & 71 & -82 & 9 & 0.23 & $18-26$ & B \\
\hline 37 & 2002-01-19 & $17: 09: 31.7$ & -1.9 & 29.6 & 33 & 4.8 & 9 & 80 & -88 & 8 & 0.32 & $13-27$ & B \\
\hline 38 & 2002-10-24 & 07:12:19.7 & -1.9 & 28.9 & 33 & 5.2 & 33 & 37 & -146 & 9 & 0.53 & $16-29$ & $\mathrm{~B}$ \\
\hline
\end{tabular}

${ }^{\mathrm{a}}$ Columns as follows: number of event, date and time of occurrence [yr-mo-day, hh:mm:ss.s], latitude, longitude, depth [km], moment magnitude $M_{W}$, strike $\phi$, dip $\delta$, rake $\lambda$, number of inverted waveform components $N_{\text {fin }}$, variance $\sigma$ (equation (1)), inverted frequency range $F_{\text {fin }}$ [mHz], quality. A more detailed list including the percentage of double couple is given as auxiliary material.

events are provided as auxiliary material and by Barth $[2007] .{ }^{1}$

\section{Earthquake Analysis}

[11] We evaluate 38 source mechanisms of earthquakes with moment magnitudes that range from $M_{W} 4.4$ to $M_{W} 5.5$ (median $M_{W} 4.8$ ) and occurred in eastern Africa from 1995 to 2002. We focus on events for which no CMT-solutions are available [e.g., Ekström et al., 2005]. To invert for the moment tensor we use a set of 14 permanent seismic stations distributed over Africa and the Indian Ocean (see inset of Figure 1).

[12] The obtained focal mechanism solutions are shown in Figure 1. Detailed inversion results of all events are given in Table 1. The obtained solutions provide a good fit between observed and modelled seismograms (average variance $\bar{\sigma}=$ $0.41 \pm .12$, average percentage of double couple $69 \% \pm 18 \%$, see auxiliary material ${ }^{1}$ ). Variance $\sigma$ increases with decreasing earthquake magnitude, restricting the studied magnitudes to $M_{W} \geq 4.4$ for eastern Africa. The frequency sensitive analysis reveals that all determined moment tensors are stable against small variations in the inverted frequency band. As variance

${ }^{1}$ Auxiliary material data sets are available at $\mathrm{ftp} / / \mathrm{ftp}$.agu.org/apend/gl/ 2007gl030359. Other auxiliary material files are in the HTML. increases by shifting the pass-band, the shapes of the moment tensors remain mostly unchanged (see Figure 2). As a further test we invert all data sets $N_{f i n}$ for a constant frequency band from $10 \mathrm{mHz}$ to $29 \mathrm{mHz}$. The results show rather high variances $\sigma$ and partially rotated mechanisms compared with the inversions of $F_{\text {fin }}$ (see Figure 2). The average number of inverted waveform traces per earthquake is nine and ranges from the predefined minimum of six to a maximum of 19 waveforms for event number $14\left(M_{W} 5.0\right)$. Some focal mechanisms of additional events analysed by inversion of five or less waveform traces tend to rotate when the frequency band is varied, since structural heterogeneities could be mapped incorrectly into the source process [e.g., Rao et al., 2002]. Therefore, we analyse only earthquakes that provide a low variance $\sigma$ and thus a good signal-to-noise ratio for at least six stations. As described by previous works, our analysis reveals that there is a clear trade-off between earthquake magnitude and the frequency range providing a sufficient signal-to-noise ratio [Braunmiller et al., 2002]. The lower the magnitude and thus the signal-to-noise ratio, the higher frequencies have to be included in the frequency range $F_{f i n}$ to obtain a stable inversion result. However, some of the 38 newly analysed earthquakes do not follow this trend, since the combination of low signal-to-noise ratio and unexplained crustal heterogeneities overlays the theoretical frequency 
dependence [Kafka and Weidner, 1979]. In order to assess the quality of the analysed events, we combine the width $\Delta F$ of the frequency range $F_{\text {fin }}$ as a measure of stability and the number $N$ of inverted waveforms $N_{\text {fin }}$ (see Table 1). Quality A $(\Delta F \geq 10$ and $N \geq 10)$ and $\mathrm{B}([\Delta F \geq 10$ and $N \leq 9]$ or $[\Delta F \leq$ 9 and $N \geq 8]$ ) are rated as reliable solutions. C qualities $(\Delta F \leq 9$ and $N \leq 7)$ are best fits, but possibly not precise in the fault plane orientations.

[13] The major part of the analysed earthquake sources are normal faulting mechanisms that are distributed over the entire study region and that agree with the general extensional regime in eastern Africa [Coblentz and Sandiford, 1994]. Beside this, we observe oblique dip-slip ruptures on the rift itself (e.g., event number 30). This supports the idea of rift-related pullapart basins as proposed by Skobelev et al. [2004]. Coblentz and Sandiford [1994] showed by modelling gravitational potential energy that compressional areas originate in the mid-ocean ridges that surround Africa. Hence, the thrust faulting events in the Mozambique Channel (event number 21) and in the Indian Ocean (event number 6) could be understood as an expression of spatial differences in potential gravitational energy. In western Africa thrust mechanisms have been described, for example, by Ayele [2002], thus event number 18 (Angola) could be interpreted as an indicator for transition from extensional tectonics in the East to a compressional regime in the West. For ten events we estimate hypocentres as deep as $33 \mathrm{~km}$ or even $42 \mathrm{~km}$ (event number 33). Similar source depths were detected for various eastern African earthquakes [e.g., Foster and Jackson, 1998; Shudofsky, 1985], indicating that in eastern Africa the seismic active zone reaches into the lower crust [Nyblade and Langston, 1995].

\section{Conclusions}

[14] The investigation of focal mechanisms of light to moderate magnitude earthquakes determined by sparse teleseismic waveform data reveals a strong dependency on the inverted frequency pass-band. Inverting a constant and relatively wide frequency band instead of a narrow one can result in rotated mechanisms. The presented frequency sensitive moment tensor inversion accounts for this uncertainty and determines a narrow pass-band within a frequency range of similar and stable moment tensors. Thus for the example of eastern Africa our analysis applies to magnitudes as low as $M_{W} 4.4$ with sparse seismic data from regional and teleseismic distances. Stable inversion results were obtained for the input of at least six waveform traces. For eastern Africa the threshold of moment tensor inversion was lowered from $M_{W} 5.2$ (median for Harvard CMT-solutions in eastern Africa) to $M_{W} 4.8$. Our analysis shows that a 1D earth model is suitable for focal mechanism determination, even though local models could improve the calculation. However, by using a 1D earth model and waveform data of the global permanent seismometer network, the described processing is easily transferable to other regions of intermediate seismicity worldwide.

[15] Acknowledgments. We thank an anonymous reviewer for valuable notes. The seismic waveforms are provided by the networks of GEOFON, GEOSCOPE, GSN and GTSN, available via IRIS datacenter Seismic data are processed with SeismicHandler [Stammler, 1993]. Figures are made using GMT [Wessel and Smith, 1998].

\section{References}

Anderson, D. L., and R. S. Hart (1978), Attenuation models of the Earth, Phys. Earth Planet. Inter., 16, 289-306.
Arvidsson, R., and G. Ekström (1998), Global CMT analysis of moderate earthquakes, $\mathrm{Mw} \geq 4.5$, using intermediate-period surface waves, Bull. Seismol. Soc. Am., 88, 1003-1013.

Ayele, A. (2002), Active compressional tectonics in central Africa and implications for plate tectonic models: Evidence from fault mechanism studies of the 1998 earthquakes in the Congo Basin, J. Afr. Earth Sci., 35, 45-50.

Barth, A. (2007), Frequency sensitive moment tensor inversion for light to moderate magnitude earthquakes in eastern Africa and derivation of the regional stress field, Ph.D. thesis, Univ. of Karlsruhe, Karlsruhe, Germany. Bernardi, F., J. Braunmiller, U. Kradolfer, and D. Giardini (2004), Automatic regional moment tensor inversion in the European-Mediterranean region, Geophys. J. Int., 157, 703-716, doi:10.1111/j.1365-246X.2004.02215.x.

Braunmiller, J., U. Kradolfer, M. Baer, and D. Giardini (2002), Regional moment tensor determination in the European-Mediterranean areaInitial results, Tectonophysics, 356, 5-22.

Coblentz, D. D., and M. Sandiford (1994), Tectonic stresses in the African plate: Constraints on the ambient lithospheric stress state, Geology, 22, 831-834.

Dziewonski, A. M., and D. L. Anderson (1981), Preliminary reference Earth model, Phys. Earth Planet. Inter., 25, 297-356.

Dziewonski, A. M., G. Ekström, J. E. Franzen, and J. H. Woodhouse (1987), Global seismicity of 1977: Centroid-moment tensor solutions for 471 earthquakes, Phys. Earth Planet. Inter., 45, 11-36.

Ekström, G., A. M. Dziewonski, N. N. Maternovskaya, and M. Nettles (2005), Global seismicity of 2002: Centroid-moment-tensor solutions for 1034 earthquakes, Phys. Earth Planet. Inter., 148, 303-326.

Engdahl, E. R., R. van der Hilst, and R. Buland (1998), Global teleseismic earthquake relocation with improved travel times and procedures for depth determination, Bull. Seismol. Soc. Am., 88, 722-743.

Fan, G., and T. Wallace (1991), The determination of source parameters for small earthquakes from a single, very broadband seismic station, Geophys. Res. Lett., 18, 1385-1388.

Foster, A. N., and J. A. Jackson (1998), Source parameters of large African earthquakes: Implications for crustal rheology and regional kinematics, Geophys. J. Int., 134, 422-448, doi:10.1046/j.1365-246x.1998.00568.x.

Giardini, D. (1992), Moment tensor inversion from mednet data: Large worldwide earthquakes of 1990, Geophys. Res. Lett., 19, 713-716.

Kafka, A. L., and D. J. Weidner (1979), The focal mechanisms and depths of small earthquakes as determined from Rayleigh-wave radiation patterns, Bull. Seismol. Soc. Am., 69, 1379-1390.

Larson, E. W. F., and G. Ekström (2001), Global models of surface wave group velocity, Pure Appl. Geophys., V158, 1377-1399.

Nyblade, A. A., and C. A. Langston (1995), East-African earthquakes below $20-\mathrm{km}$ depth and their implications for crustal structure, Geophys. J. Int., 121, 49-62.

Pinar, A., K. Kuge, and Y. Honkura (2003), Moment tensor inversion of recent small to moderate sized earthquakes: Implications for seismic hazard and active tectonics beneath the Sea of Marmara, Geophys. J. Int., 153, 133-145.

Rao, N. P., T. Tsukuda, M. Kosuga, S. C. Bhatia, and G. Suresh (2002), Deep lower crustal earthquakes in central India: Inferences from analysis of regional broadband data of the 1997 May 21, Jabalpur earthquake, Geophys. J. Int., 148, 132-138.

Shudofsky, G. N. (1985), Source mechanisms and focal depths of East African earthquakes using Rayleigh-wave inversion and body-wave modelling, Geophys. J. R. Astron. Soc., 83, 563-614.

Skobelev, S. F., M. Hanon, J. Klerkx, N. N. Govorova, N. V. Lukina, and V. G. Kazmin (2004), Active faults in Africa: A review, Tectonophysics, 380, $131-137$.

Stammler, K. (1993), SeismicHandler: Programmable multichannel data handler for interactive and automatic processing of seismological analyses, Comput. Geosci., 19, 135-140.

Stich, D., C. Ammon, and J. Morales (2003), Moment tensor solutions for small and moderate earthquakes in the Ibero-Magrebh region, J. Geophys. Res., 108(B3), 2148, doi:10.1029/2002JB002057.

Stutzmann, E., G. Roult, and L. Astiz (2000), GEOSCOPE station noise levels, Bull. Seismol. Soc. Am., 90, 690-701.

Wessel, P., and W. H. F. Smith (1998), New, improved version of Generic Mapping Tools released, Eos Trans. $A G U, 79,579$.

Woodhouse, J. H. (1988), The calculation of eigenfrequencies and eigenfunctions of the free oscillations of the Earth and the Sun, in Seismological Algorithms, edited by D. J. Doornbos, pp. 321-370, Academic Press, London.

A. Barth and F. Wenzel, Geophysical Institute, University of Karlsruhe, Hertzstr. 16, 76187 Karlsruhe, Germany. (andreas.barth@gpi. uni-karlsruhe.de)

D. Giardini, Swiss Seismological Service, Institute of Geophysics, ETH Zürich, Schafmattstr. 30, 8093 Zürich, Switzerland. 